\title{
Continuous Glucose Monitoring for Underserved and Minority Patients with Type 2 Diabetes in an Interprofessional Internal Medicine Clinic
}

\author{
Arden Bui, PharmD Candidate ${ }^{1}$; Jennifer Kim, PharmD, BCPS, BCACP, BC-ADM, CDE, CPP1,2 \\ ${ }^{1}$ University of North Carolina Eshelman School of Pharmacy, Chapel Hill, NC \\ ${ }^{2}$ Moses Cone Internal Medicine Clinic, Cone Health, Greensboro, NC
}

\begin{abstract}
Objective: Evaluate the impact of interprofessional continuous glucose monitoring (CGM) on glycemic control in underserved and minority patients with type 2 diabetes mellitus (T2DM)

Methods: The IRB-approved, retrospective quasi-experimental study was conducted between August 2018 and August 2019 at an internal medicine residency clinic. Adult patients with diagnosed T2DM, an indication for CGM (hyperglycemia, hypoglycemia, and/or glycemic variability), and at least 10 consecutive days of CGM data were included. Patients who were pregnant and/or missed one or more clinic appointments were excluded. Patients were followed weekly over a 14-day period during which data from the CGM sensor were downloaded and interpreted by the interprofessional team. Interventions with shared decision-making were made at each visit. Results: Fifty-five patients were included, with a mean age of $61 \pm 11.2$ years, $86 \%$ were of non-white race, and $56 \%$ were female. Average blood glucose levels decreased from $208.39 \mathrm{mg} / \mathrm{dL}$ at week 1 to $190.74 \mathrm{mg} / \mathrm{dL}$ at week 2, a $17.65 \mathrm{mg} / \mathrm{dL}$ reduction (p=0.0281). Time within target range $(70-180 \mathrm{mg} / \mathrm{dL}$ ) increased by $6.16 \%(p=0.0038)$, while time above range $(>180 \mathrm{mg} / \mathrm{dL}) \mathrm{decreased}$ by $5.5 \%(p$ $=0.0168$ ). Average number of hypoglycemic events (readings $<70 \mathrm{mg} / \mathrm{dL}$ ) did not change significantly from week 1 to week 2 (2.94 vs. $3.64, p>0.05)$. Of the 66 interventions made after week 1,33\% were made by the pharmacy team, 56\% were made by the attending or resident physicians, and $11 \%$ were made by the dietician. Of 59 interventions made after week 2, 32\% were made by the pharmacy team, $49 \%$ were made by the attending or resident physicians, and 19\% were made by the dietician.

Conclusions: The interprofessional collaborative CGM service was associated with improvements in glucose control based on increased time within target range and reduced hyperglycemia, without increasing the risk of hypoglycemia or medication burden.
\end{abstract}

Keywords: Interprofessional, continuous glucose monitoring, diabetes, pharmacy education

\section{Background}

Continuous glucose monitoring (CGM) is a valuable tool to accurately assess glycemic control in patients with diabetes. CGM utilization provides healthcare professionals with better insight into glucose trends and allows more personalized interventions based on pharmacotherapy, diet, and physical activity. ${ }^{1}$ Recent studies have demonstrated the clinical benefits of CGM by reducing glycated hemoglobin A1c (HbA1c) and blood glucose levels in patients with type 1 diabetes (T1DM) and type 2 diabetes (T2DM). ${ }^{2,3}$

Though CGM has demonstrated benefit for a variety of populations, its utility in interprofessional teaching settings and for underserved patients has not been sufficiently explored. An interprofessional practice model integrates different healthcare professionals into a care setting to provide better services and support for patients. ${ }^{4,5}$ For instance, dieticians offer evidence-based nutrition recommendations and dietary advice to patients with diabetes. Pharmacists can help guide treatment decisions by selecting the medication that maximizes

Corresponding author: Jennifer Kim, PharmD, BCPS, BCACP, BC-ADM, CDE, CPP

Email: jen.kim@conehealth.com safety, minimizes side effects, and benefits other comorbidities (e.g., heart failure, chronic kidney disease). Pharmacists can also assist patients with medication adherence and drug affordability. ${ }^{6}$ Thus, interprofessional collaboration maximizes patient outcomes by averting preventable errors, strengthening relationships with other disciplines, and reducing healthcare costs. ${ }^{4,5}$ A cohort study evaluating the efficacy of interprofessional practice models showed that collaborative care was associated with statistically significant reductions in hospitalizations, ED visits, $\mathrm{HbA1c}$, and patient charges over a 1year period. ${ }^{5}$

In patients with diabetes, low-income earners have a greater risk of hospitalizations for acute diabetes-related complications and death than high-income earners. ${ }^{7}$ To date, only 1 published CGM study in a low-income population was identified, in which CGM did not show significant reductions in HbA1c among lowincome, high-risk Latino patients with type 1 diabetes. ${ }^{8}$ However, the study was underpowered, did not implement an interprofessional practice model, and the duration of the study was too brief to assess the potential benefits of CGM. ${ }^{7}$ Many patients in the internal medicine residency clinic are lowincome and under-insured or uninsured. The purpose of this research was to describe an interprofessional CGM service for underserved T2DM patients in an internal medicine residency clinic in Greensboro, NC. 


\section{Methods}

A new CGM service was implemented in 2018 at an internal medicine residency clinic that provides primary care for adult patients regardless of financial status, with many patients estimated to fall within $200 \%$ of the Federal Poverty Level. ${ }^{9}$ The CGM service involved shared decision-making among attending and resident physicians, pharmacists, dieticians, and health professional students. The CGM process (appointments, follow up plans, billing) was developed by a physician leader, pharmacist, and dietician. The clinic director funded and managed CGM supply inventory. The dietician implemented the CGM software and provided staff education on the CGM process and billing.

Patients were offered to participate in the CGM service based on clinical judgement by the team (concern for hypoglycemia, hyperglycemia, or glycemic variability). Inclusion criteria were age 18 years or older, T2DM diagnosis, participation in the clinic CGM service, and at least 10 consecutive days of CGM data available. Ten days was determined to allow ample time for clinical evaluation of response to therapy changes. Exclusion criteria included pregnancy, less than 10 days of consecutive CGM data, and/or missed one or more clinic appointments. For each patient, 3 appointments were scheduled, each spaced one week apart. The first appointment (day 1) involved CGM placement and education (billing codes: CPT $99211-5$ in addition to G95250 for CGM placement); the second and third appointments (week 1 and week 2 ) involved data download and therapy changes with education (billing codes: CPT 99211-5 in addition to G95251 for CGM analysis, interpretation, and report). ${ }^{10}$ The CGM sensor was removed at the third visit. The dietician, pharmacist, or clinic phlebotomists downloaded and shared CGM data with the physician seeing the patient that day. The dietician or pharmacist implemented therapy changes in collaboration with, or provided recommendations to, the attending or resident physicians. The pharmacist managed inventory for diabetes sample medications and assisted with overcoming access barriers to support real-time therapy changes and allow for evaluation of response to therapy and titration. Pharmacy residents and students on rotation participated in all aspects of the CGM process under pharmacist preceptor guidance.

The primary outcomes included change in average blood glucose from the end of week 1 to end of week 2, mean percentage of time blood glucose was above target range $(>180$ $\mathrm{mg} / \mathrm{dl})$, within target range $(70-180 \mathrm{mg} / \mathrm{dl})$, and below target range $(<70 \mathrm{mg} / \mathrm{dl})$ at the end of week 1 and week 2 , and preand post-CGM number of diabetes medications. Secondary outcomes included number of hypoglycemic events (readings below $70 \mathrm{mg} / \mathrm{dl}$ ), mean pre- and post-CGM HbA1c, and number of interventions made by the interprofessional team at each week. Only diabetes-specific interventions were accounted for during this study. Pre- and post-HbA1c levels were recorded within 3 months prior and 3 months after the 2-week CGM service, respectively. Interventions included adjusting medication doses, adding agents, stopping agents, and/or providing medication or lifestyle education. Pharmacotherapy may have been changed due to poor glycemic control, nonadherence, cost, side effects, inadequate response, high medication burden, and/or clinical contraindication. An exploratory outcome included the number of interventions made by each healthcare professional. Data was collected through chart review via the electronic medical record and analyzed using paired t-tests with $\mathrm{JMP}^{\oplus}$ and descriptive statistics. $^{11}$

\section{Results}

A total of 55 patients were included, with a mean age of $61 \pm 11.2$ years, $85 \%$ were non-Caucasian or non-white race, and $56 \%$ were female. Baseline characteristics are summarized in Table 1.

For primary outcomes, average blood glucose levels decreased from $208.39 \mathrm{mg} / \mathrm{dL}$ at week 1 to $190.74 \mathrm{mg} / \mathrm{dL}$ at week 2, a $17.65 \mathrm{mg} / \mathrm{dL}$ reduction, $(\mathrm{p}=0.0281$ ) (see Table 2). The percentage of time within target range $(70-180 \mathrm{mg} / \mathrm{dl})$ increased by $6.16 \%(p=0.0038)$, and the percentage of time above $180 \mathrm{mg} / \mathrm{dl}$ decreased by $5.5 \%$ ( $p=0.0168)$. The average percentage of time blood glucose was below $70 \mathrm{mg} / \mathrm{dL}$ did not change significantly $(p=0.99)$. Number of diabetes medications did not change significantly pre-and post-CGM (2.56 v. 2.42, p > 0.05). Weekly clinical outcomes based on indication for CGM are shown in Table 3. All three subgroups (glycemic variability, hypoglycemia, and hyperglycemia) demonstrated numerical increases in time within target range.

For secondary outcomes, the average number of hypoglycemic events did not significantly change from week 1 to week 2 (2.94 vs. 3.64, $p>0.05)$. Mean HgbA1c values decreased from $9.09 \%$ to $8.46 \%$, a $0.63 \%$ difference, $(p=0.0092)$. A summary of interventions made are shown in Table 4 . Of 66 interventions made at week 1, 24.24\% had medication doses increased, $16.67 \%$ had medication doses reduced, $6.06 \%$ received additional medications, $13.64 \%$ had medications stopped, $15.15 \%$ had medications switched, and $24.24 \%$ received diabetes education. Four patients did not receive any interventions at week 1 . Of 59 interventions made at week 2, $27.59 \%$ had medication doses increased, $12.07 \%$ had medication doses reduced, $12.07 \%$ received additional medications, $1.72 \%$ had medications stopped, $10.34 \%$ had medications switched, and $36.21 \%$ received diabetes education. Five patients did not receive any interventions at week 2 .

For the exploratory outcome, of the 66 interventions made at week $1,33 \%$ were made by the pharmacy team, $56 \%$ were made by attending or resident physicians, and $11 \%$ were made by the dietician. Of 59 interventions made at week 2, 32\% were made by the pharmacy team, $49 \%$ were made by the attending or resident physicians, and $19 \%$ were made by the dietician. 


\section{Discussion}

The results of this study align with previous studies that employing CGM can improve glycemic control without significantly increasing the risk of hypoglycemia. For patients with hypoglycemia, CGM can reduce time spent in hypoglycemia, with improved time within range. ${ }^{12}$ Most studies have included patients with T1DM; data are less clear for T2DM. Studies in T2DM have demonstrated improved glycemic control with no significant impact on hypoglycemia. ${ }^{13}$ In our study T2DM patients, time above range and time within target range significantly improved from week 1 to week 2 , while time below range did not change significantly.

Research is lacking for use of CGM in an interprofessional setting and in underserved populations. ${ }^{14}$ Improving healthcare access for underserved patients can minimize disparities and risk for diabetes-related complications. ${ }^{15-18}$ To our knowledge, only 1 CGM study in a low-income population has been published, which included adults with T1DM. ${ }^{19}$ According to Sequeira et al., over $80 \%$ of patients reported that CGM helped to prevent hypoglycemia and improved hypoglycemia management. There was no significant HgbA1c improvement, potentially due to a relatively short duration of about 22 weeks, in addition to literacy and resource constraints.

This study was aimed to assess the impact of an interprofessional CGM service on glycemic control in underserved patients with T2DM. Our results illustrate the contributions of team members involved and the potential benefits of a team-based initiative utilizing newer technology and providing a progressive learning opportunity for trainees. Interprofessional collaboration enhances patient safety, improves patient care quality, supports high-quality education for healthcare professionals in training, and is a core standard of medical residency training. ${ }^{20,21}$

This study had several limitations. First, the study was underpowered to infer causality due to small sample size. Second, interventions were evaluated retrospectively on the electronic medical record and credited to the provider(s) who inputted the intervention(s). This method did not account for any verbal or undocumented interprofessional interactions that may have occurred during the clinical decision-making process. This may have led to an under- or overestimation of the number of interventions implemented by each health professional. Third, the study did not evaluate medication adherence. Pharmacologic interventions were recorded regardless of whether the patient complied. Behavioral changes may have also affected glycemic control, as patients were aware of the round-the-clock glucose monitoring feature of CGM. We also did not compare this intervention to usual care, conducted the study over a short timeframe (3 months before and after the 2week intervention), and did not evaluate return on investment which would be of interest for long-term sustainability (e.g., offsetting the clinic cost of CGM equipment and potential need for additional staffing with revenue from appointment billing, reduced acute care visits and hospitalizations, and improved performance metrics). Lastly, evaluation of patient feedback and satisfaction of the CGM service may help guide future quality improvement projects.

To meet the needs of a growing diabetic population, especially in populations with limited access who are experiencing increased incidences of diabetes diagnoses and related complications, expanding access to newer strategies and a variety of health disciplines is paramount to maximizing positive outcomes. Team-based clinical decision-making can be supported by providing insight into how glucose-lowering medications, diet, and exercise influence glycemic control. Data from CGM may then be used by the healthcare team to coordinate an appropriate plan that addresses patient-specific needs in pharmacotherapy and diabetes education.

\section{Conclusions}

The interprofessional CGM service was associated with improvements in glycemic control based on decreased average blood glucose and increased time within range without increasing the risk of hypoglycemia or medication burden. The team-based approach to diabetes management at the internal medicine teaching clinic allowed practitioners of various disciplines to take an active role in a patient's care and improve health outcomes.

\section{Acknowledgement: none \\ Funding/support: none \\ Conflicts of interest: none}

Treatment of human subjects: IRB review/approval required and obtained

\section{Resources}

1. Shrivastav, M, Gibson, W, et al. Type 2 Diabetes Management in Primary Care: The role of retrospective continuous glucose monitoring. Diabetes Spectrum. Aug 2018, 31 (3) 279-287.

2. Ajjan RA, Jackson N, Thomson SA. Reduction in HbA1c using professional flash glucose monitoring in insulintreated type 2 diabetes patients managed in primary and secondary care settings: A pilot, multicentre, randomised controlled trial. Diab Vasc Dis Res. 2019;16(4):385-395.

3. Rodbard D. Continuous Glucose Monitoring: A Review of Recent Studies Demonstrating Improved Glycemic Outcomes. Diabetes Technol Ther. 2017;19(S3):S25S37.

4. Gucciardi E, Espin S, Morganti A, Dorado L. Exploring interprofessional collaboration during the integration of diabetes teams into primary care. BMC Fam Pract. 2016;17:12. 
5. Guck TP, Potthoff MR, Walters RW, et al. Improved Outcomes Associated With Interprofessional Collaborative Practice. Ann Fam Med. 2019;17(Suppl 1):S82.

6. MacDonald J. Pharmacists play an essential role in a diabetes care team. Drug Topics. 2020;164(2):7-8.

7. Rawshani A, Svensson A, Zethelius B, et al. Association Between Socioeconomic Status and Mortality, Cardiovascular Disease, and Cancer in Patients With Type 2 Diabetes. JAMA Intern Med. 2016;176(8):1146-1154.

8. Sequeira PA, Montoya L, Ruelas V, et al. Continuous glucose monitoring pilot in low-income type 1 diabetes patients. Diabetes Technol Ther. 2013;15:855-858.

9. Assistant Secretary for Planning and Evaluation. Poverty Guidelines. United States Department of Health and Human Services. 2020 Jan 15. https://aspe.hhs.gov/poverty-guidelines. Accessed August 13, 2020.

10. American Association of Diabetes Educators. Professional continuous glucose monitoring implementation playbook. 2019. http://assets.aanp.org/documents/2019/Prof_CGM_ Playbook_AADE_AANP.pdf. Accessed October 28, 2020.

11. $\mathrm{JMP}^{\circledR}$, Version 15.1.0, SAS Institute Inc., Cary, NC, 1989-2019.

12. Maiorino MI, Signoriello S, Maio A, et al. Effects of continuous glucose monitoring on metrics of glycemic control in diabetes: a systematic review with metaanalysis of randomized controlled trials. Diabetes Care. 2020;43:1146-1156.

13. Mark C, Le QA. The effectiveness of continuous glucose monitoring in patients with type 2 diabetes: a systematic review of literature and meta-analysis. Diabetes Technol Ther. 2018;20:613-621.
14. American Diabetes Association. Diabetes technology: Standards of medical care in diabetes -2020. Diabetes Care. 2020;43(Supplement 1):S77-S88.

15. Centers for Disease Control and Prevention. Addressing healthcare disparities in diabetes. Diabetes. Last updated April 15, 2019. https://www.cdc.gov/diabetes/disparities.html. Accessed June 16, 2020.

16. Chow EA, Foster H, Gonzalez V, Mclver L. The disparate impact of diabetes on racial/ethnic minority populations. Clin Diabetes. 2012;30(3):130-133.

17. Piccolo RS, Subramanian SV, Pearce N, Florez JC, McKinlay JB. Relative contributions of socioeconomic, local environment, psychosocial, lifestyle/behavioral, biophysiological, and ancestral factors to racial/ethnic disparities in type 2 diabetes. Diabetes Care. 2016;39(7):1208-1217.

18. Peterson K, Anderson J, Boundy E, Ferguson L, McLeery E, Waldrip K. Mortality disparities in racial/ethnic minority groups in the Veterans Health Administration: An evidence review and map. Am J Public Health. 2018;108(3):e1-e11.

19. Sequeira PA, Montoya L, Ruelas V, et al. Continuous glucose monitoring pilot in low-income type 1 diabetes patients. Diabetes Technol Ther. 2013;15:855-858.

20. Interprofessional Education Collaborative Expert Panel. Core competencies for interprofessional collaborative practice: Report of an expert panel. 2011. Washington, D.C.: Interprofessional Education Collaborative.

21. Accreditation Council for Graduate Medical Education (ACGME). The Program Directors' Guide to the Common Program Requirements (Residency). 2020. https://www.acgme.org/Portals/0/PFAssets/Program Resources/PDGuideResidency.pdf. Accessed August 18, 2020. 
Table 1: Baseline characteristics

\begin{tabular}{|l|r|}
\hline Total patients, $\mathrm{n}$ & 55 \\
\hline Age, mean years (SD) & $61(11.2)$ \\
\hline Males, $\mathrm{n}$ & $24(44 \%)$ \\
\hline Females, $\mathrm{n}$ & $31(56 \%)$ \\
\hline Caucasian or White, $\mathrm{n}$ & $8(15 \%)$ \\
\hline African American or Black, $\mathrm{n}$ & $43(78 \%)$ \\
\hline Hispanic, $\mathrm{n}$ & $2(4 \%)$ \\
\hline Asian, $\mathrm{n}$ & $1(2 \%)$ \\
\hline Mixed or other, $\mathrm{n}$ & $1(2 \%)$ \\
\hline Pre-CGM A1c $<7 \%, \mathrm{n}$ & $12(22 \%)$ \\
\hline Pre-CGM A1c $7-9 \%, \mathrm{n}$ & $19(35 \%)$ \\
\hline Pre-CGM A1c $>9 \%, \mathrm{n}$ & $24(44 \%)$ \\
\hline Glycemic variability, $\mathrm{n}$ & 13 \\
\hline Hypoglycemia, $\mathrm{n}$ & 20 \\
\hline Hyperglycemia, $\mathrm{n}$ & 22 \\
\hline
\end{tabular}

Table 2: Primary endpoints

\begin{tabular}{|l|c|c|c|}
\hline Endpoints & CGM week \#1 & CGM week \#2 & $\begin{array}{c}\text { P value } \\
\text { (95\% CI) }\end{array}$ \\
\hline Average blood glucose, mg/dL (SD) & $208.39(58.7)$ & $190.74(55.5)$ & $\begin{array}{c}0.028 \\
\text { (-33.35 to }-1.97)\end{array}$ \\
\hline Time with glucose $<70 \mathrm{mg} / \mathrm{dL}$, mean \% (SD) & $2.95(4.0)$ & $5.04(8.2)$ & $\begin{array}{c}0.99 \\
\text { (-2.2 to 2.19) }\end{array}$ \\
\hline Time with glucose 70-180 mg/dL, mean \% (SD) & $45.22(31.2)$ & $51.38(31.8)$ & $\begin{array}{c}0.0038 \\
\text { (2.08 to } 10.24)\end{array}$ \\
\hline Time with glucose >180 mg/dL, mean \% (SD) & $49.79(34.7)$ & $44.3(34.6)$ & $\begin{array}{c}0.0168 \\
\text { (-9.94 to }-1.03)\end{array}$ \\
\hline Number of DM medications, mean (SD) & Pre-CGM & Post-CGM & P value \\
\hline
\end{tabular}

$\mathrm{Cl}$, confidence interval; A1C, glycated hemoglobin; DM, diabetes mellitus; SD, standard deviation

Table 3: Pre-post comparisons associated with CGM based on indication

\begin{tabular}{|l|c|c|c|}
\hline & $\begin{array}{c}\text { Glycemic variability } \\
(\mathbf{n = 1 3 )}\end{array}$ & $\begin{array}{c}\text { Hypoglycemia } \\
(\mathbf{n = 2 0 )}\end{array}$ & $\begin{array}{c}\text { Hyperglycemia } \\
\text { (n=22) }\end{array}$ \\
\hline Mean blood glucose week \#1, mg/dL (SD) & $265.2(99.1)$ & $153(56.1)$ & $228.7(95.5)$ \\
\hline Mean blood glucose week \#2, mg/dL (SD) & $226.5(84.4)$ & $153(62.9)$ & $205.1(85.8)$ \\
\hline Time within range week \#1, mean \% (SD) & $27.9(29.8)$ & $63.2(24.1)$ & $37.0(29.8)$ \\
\hline Time within range week \#2, mean \% (SD) & $36.3(30.5)$ & $67.9(26.0)$ & $44.2(32.4)$ \\
\hline Pre-CGM A1C, mean \% (SD) & $9.8(1.7)$ & $7.7(1.9)$ & $9.8(2.3)$ \\
\hline Post-CGM A1c, mean \% (SD) & $9.2(1.4)$ & $7.6(2)$ & $8.8(2)$ \\
\hline
\end{tabular}

CGM, continuous glucose monitor; SD, standard deviation

Table 4: Interventions made by interprofessional team at week 1 and week 2

\begin{tabular}{|l|c|c|}
\hline Intervention type & Week 1, $\mathbf{n}(\mathbf{\%})$ & Week 2, $\mathbf{n}(\%)$ \\
\hline Increased medication dose & $16(24.2)$ & $16(27.6)$ \\
\hline Decreased medication dose & $11(16.7)$ & $7(12.1)$ \\
\hline Added medication & $4(6.1)$ & $7(12.1)$ \\
\hline Stopped medication & $9(13.6)$ & $1(1.7)$ \\
\hline Switched medication & $10(15.2)$ & $6(10.3)$ \\
\hline Medication or lifestyle education & $16(24.2)$ & $21(36.2)$ \\
\hline Interventions made by health profession & Week 1, $\mathbf{n}(\mathbf{\%})$ & $\mathbf{W e e k ~ 2 , ~} \mathbf{n}(\mathbf{\%})$ \\
\hline Pharmacy team & $21(33)$ & $19(32)$ \\
\hline Attending or resident physicians & $37(56)$ & $29(49)$ \\
\hline Dietician & $8(11)$ & $11(19)$ \\
\hline
\end{tabular}

